\title{
Cholesterol oxidase from Bordetella species promotes irreversible cell apoptosis in lung adenocarcinoma by cholesterol oxidation
}

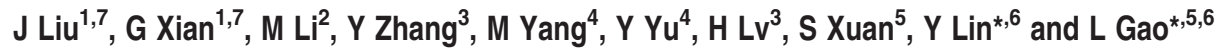

Cholesterol oxidase (COD), an enzyme catalyzing the oxidation of cholesterol, has been applied to track the distribution of membrane cholesterol. Little investigations about the effect of COD on tumor cells have been performed. In the present study, we provided evidence that COD from Bordetella species (COD-B), induced apoptosis of lung cancer cells in vitro and in vivo. COD-B treatment inhibited Akt and ERK1/2 phosphorylation in dose- and time-dependent manner, which was not reversed and was even aggravated by cholesterol addition. Further investigation indicated that COD-B treatment promoted the generation of reactive oxygen species (ROS) and that cholesterol addition further elevated ROS levels. Moreover, COD-B treatment resulted in JNK and p38 phosphorylation, downregulation of $\mathrm{Bcl}-2$, upregulation of Bax, activated caspase-3 and cytochrome $C$ release, which likely responded to freshly produced hydrogen peroxide that accompanied cholesterol oxidation. Catalase pretreatment could only partially prevent COD-B-induced events, suggesting that catalase inhibited $\mathrm{H}_{2} \mathrm{O}_{2}$-induced signal transduction but had little effect on signal pathways involved in cholesterol depletion. Our results demonstrated that COD-B led to irreversible cell apoptosis by decreasing cholesterol content and increasing ROS level. In addition, COD-B may be a promising candidate for a novel anti-tumor therapy.

Cell Death and Disease (2014) 5, e1372; doi:10.1038/cddis.2014.324; published online 14 August 2014

Cholesterol is an essential component of the plasma membrane in eukaryotic cells and has an important role in maintaining structure integrity, receptor function, dynamics and ion channels in the plasma membrane. ${ }^{1-3}$ Cholesterol is a critical constituent for the formation of lipid rafts. ${ }^{4}$ Lipid rafts are plasma membrane microdomains locating abundant signaling molecules, such as caveolin-1 protein and epidermal growth factor receptor (EGFR). ${ }^{5}$ These molecules conduct a series of cellular functions, including cell proliferation and apoptosis. ${ }^{6}$ Because cholesterol has a bridging role in liquid-ordered rafts by binding tightly to the sphingolipids with saturated hydrocarbon chains, modification or depletion of membrane cholesterol is speculated to perturb the properties of lipid rafts. ${ }^{7,8}$ Several studies have demonstrated that the depletion of membrane cholesterol led to the disruption of lipid rafts and dissociation of signaling molecules from lipid rafts, which generated aberrant signal transductions and disturbed cellular functions. ${ }^{9}$ Therefore, membrane cholesterol concentration is accurately regulated. ${ }^{10}$

Cholesterol metabolism is disorganized in various tumors, such as prostate, lung, acute myeloid leukemia and breast cancer and especially in chemoresistant tumors. ${ }^{11-15}$ Solid tumors accumulate more cholesterol compared with normal tissue, which contributes to the proliferation, differentiation and migration of tumor cell. ${ }^{16,17}$ The elevated content of membrane cholesterol modulates the activation of cellular surface receptors, such as EGFR. ${ }^{18,19}$ Several reports have demonstrated that EGFR is upregulated in most malignant cells and stimulates the proliferation of cells by promoting the downstream activation of protein kinase B (Akt). ${ }^{20-22}$ Depletion of cholesterol from plasma membrane induces Akt inactivation and cell death. ${ }^{23,24}$ Furthermore, the alteration of membrane cholesterol also affects the expression of the B-cell lymphoma/leukemia-2 (BCL-2) family members. ${ }^{21,25}$ Therefore, it has been proposed that membrane cholesterol could potentially be a therapeutic target for tumors.

The modification of membrane cholesterol can be mainly performed by methyl-beta-cyclodextrin $(\mathrm{M} \beta \mathrm{CD})$, statin and filipin. ${ }^{9,21}$ Previous reports have indicated that $\mathrm{M} \beta \mathrm{CD}$ could take up cholesterol into the internal hydrophobic pocket to form a $\mathrm{M} \beta \mathrm{CD}$-cholesterol complex and that the hydrophilic surface promoted the complex to dissolve in the aqueous phase. ${ }^{26}$ Statins serve as competitive inhibitors to 3-hydroxy3-methylglutaryl CoA reductase (HMG-CoA) to block

\footnotetext{
${ }^{1}$ Department of General Surgery, Shandong Provincial Hospital Affiliated to Shandong University, Jinan 250021, China; ${ }^{2}$ Department of Cardiology, Shandong Provincial Hospital Affiliated to Shandong University, Jinan 250021, China; ${ }^{3}$ Department of Obstetrics, Shandong Provincial Hospital Affiliated to Shandong University, Jinan 250021, China; ${ }^{4}$ Department of Ultrasound, Shandong Provincial Hospital Affiliated to Shandong University, Jinan 250021, China; ${ }^{5}$ Department of Endocrinology, Shandong Provincial Hospital Affiliated to Shandong University, Jinan, Shandong Province 250021, China and ${ }^{6}$ Department of Center Laboratory, Shandong Provincial Hospital Affiliated to Shandong University, Jinan 250021, China

${ }^{*}$ Corresponding author: Y Lin or L Gao, Department of Center Laboratory, Shandong Provincial Hospital Affiliated to Shandong University, Jingwuweiqi Road 324\#, Jinan 250021, China. Tel.: +86 531 68776912; Fax: +86 531 87906016; E-mail: sphyanlianglin@yahoo.com (YL) or Tel.: +86 531 85186910; Fax: +86 531 87068707; E-mail: gaoling1@medmail.com.cn (LG)

${ }^{7}$ These authors contributed equally to this work.

Abbreviations: Akt, protein kinase B; BCL-2, B-cell lymphoma/leukemia-2; COD, cholesterol oxidase; COD-B, cholesterol oxidase from Bordetella species; EGFR, epidermal growth factor receptor; ERK1/2, extracellular signal-regulated kinase 1/2; HMG-CoA, 3-hydroxy-3-methylglutaryl CoA reductase; M $\beta C D$, methyl-betacyclodextrin; ROS, reactive oxygen species

Received 19.3.14; revised 24.6.14; accepted 26.6.14; Edited by A Stephanou
} 
synthesis of cholesterol. Filipin, as a macrolide polyene antibiotic, binds specifically to cholesterol and causes the disruption of lipid rafts. ${ }^{27} \mathrm{~A}$ common factor shared by the above three agents is that the cholesterol alteration caused by each of them can be reversed by the supplement of exogenous cholesterol. In view of high cholesterol in the modern diet, the effect of $\mathrm{M} \beta \mathrm{CD}$, statin and filipin on tumors would be attenuated, although prolonged suppression of cholesterol synthesis reduces the risk of advanced prostate cancer. $^{28}$

Cholesterol oxidase (COD) is a flavoprotein from microorganisms that catalyzes the oxidation of cholesterol to 4-cholesten-3-one with the reduction of oxygen to hydrogen peroxide. $^{29}$ COD can convert membrane cholesterol to 4-cholesten-3-one and can inhibit the formation of lipid rafts. ${ }^{30}$ Different from other cholesterol-depleting agents, COD disrupts lipid rafts by displacing cholesterol with 4-cholesten-3-one. However, because the action of COD is dependent on the microenvironment of membrane cholesterol, such as the phospholipid composition, cholesterol content and ionic strength, COD does not attack all cell types. So far, despite the fact that COD has been well known as a lipid raft destroyer, few investigations have been conducted to directly determine the impact of COD on signal transduction in cancer.

In this study, we investigated whether COD purified from Bordetella species (COD-B) could suppress the growth of a lung adenocarcinoma cell line by its effect on membrane cholesterol. We provide the evidence that COD-B induces apoptosis in lung adenocarcinoma cells by catalyzing oxidation of membrane cholesterol and elevating reactive oxygen species (ROS) levels. Moreover, COD-B-induced apoptosis is not reversed and is aggravated by cholesterol supplementation.

\section{Results}

COD-B catalyzed the oxidation of membrane cholesterol in lung adenocarcinoma cells. Previous studies have demonstrated that membrane cholesterol could be oxidized by COD. ${ }^{31,32}$ In this investigation, we examined whether COD-B oxidized membrane cholesterol in lung adenocarcinoma cells. We first measured the cholesterol alteration probed by the sterol-specific dye filipin in whole cells. The results showed that COD-B treatment induced membrane cholesterol levels despite cholesterol repletion by the exogenous cholesterol (Figure 1A). Consistent with cholesterol staining, TLC analysis confirmed that treatment of A549 cells with COD-B caused oxidation of cholesterol (Figure $1 \mathrm{Ba}$ ). The product held functional groups visualized by UV irradiation (Figure 1Bb). Mass spectrum (MS) analysis verified that the molecular weight $\left(M_{r}\right)$ of the product was 384.7 consistent with 4-cholesten-3-one by Figure 1D. Exposure of A549 cells to $1 \mathrm{U} / \mathrm{ml}$ COD-B for $6 \mathrm{~h}$ resulted in the oxidation of nearly $60 \%$ of the cholesterol, and cholesterol addition could partially prevent the oxidation but did not affect the formation of 4-cholesten-3-one (Figure 1C). Taken together, the oxidation of membrane cholesterol was caused by exposure of A549 cells to COD-B (Figure 1E) and could not be completely restored by cholesterol addition.
COD-B-induced lung adenocarcinoma cell apoptosis. Because of the important role of membrane cholesterol in signal transduction, we tested the effect of COD-B on A549 and SPC-A-1 cells' viabilities. After exposure to COD-B for $24 \mathrm{~h}, \mathrm{~A} 549$ and SPC-A-1 cells' viabilities were significantly inhibited by COD-B with $\mathrm{IC}_{50}$ of $0.34 \pm 0.04 \mathrm{U} / \mathrm{ml}$ and $0.52 \pm 0.03 \mathrm{U} / \mathrm{ml}$, respectively (Figure 2a). Exposure of A549 and SPC-A-1 cells to $1.0 \mathrm{U} / \mathrm{ml}$ COD-B for $6 \mathrm{~h}$ induced cell death in approximately 70 and $50 \%$ of the cells (Figure 2b). After treatment with COD-B, the cells were progressively more circular shaped with increasing concentrations of COD-B (Figure 2c). Because the process of apoptosis is accompanied by morphological changes, ${ }^{33,34}$ and membrane cholesterol was implicated in pro-apoptotic and anti-apoptotic signaling pathways, we assessed whether COD-B influenced the activation of Akt and extracellular signal-regulated kinase 1/2 (ERK1/2) for cell survival, as well as caspase-3 for cell apoptosis. As shown in Figure $2 \mathrm{~d}$, with increasing doses, COD-B gradually suppressed the phosphorylation of Akt and ERK1/2 and activated caspase-3. To verify whether the COD-B-induced Akt and ERK1/2 inactivation could be reversed by supplying cholesterol, cells were treated with COD-B for $6 \mathrm{~h}$ followed by incubation with $1 \mathrm{mM}$ cholesterol for $1 \mathrm{~h}$, and the phosphorylation of Akt and ERK1/2 was determined by western blotting. Figure $2 e$ illustrates that cholesterol supplementation slightly restored the activation of Akt and ERK1/2, whereas we noted an increase in the accumulation of activated caspase-3. TUNEL staining showed that the exposure to COD-B led to extensive DNA damage with increasing doses of COD-B (Figure 3a), which confirmed COD-B-induced apoptosis. Internucleosomal degradation of the genome DNA, another biochemical hallmark of apoptosis, was further analyzed by agarose electrophoresis. As shown in Figure $4 b$, exposure of cells to COD-B caused significant degradation of the genome DNA with increasing time of treatment, which was further aggravated by cholesterol addition. Inconsistent with the results in Figure 1E, cholesterol addition did not prevent cell apoptosis but even slightly promoted cell apoptosis, which was further verified by measuring Annexin $\mathrm{V}$ binding to cell membranes using flow cytometer analysis (Figure 3b). It was perplexing that cholesterol addition slightly activated Akt and ERK1/2, whereas it did not prevent cell apoptosis. To explain these results, we tested whether ROS had a role in COD-B-induced apoptosis.

The ROS-mitochondrial apoptotic pathway was involved in COD-B-induced apoptosis. Given that hydrogen peroxide was generated along with cholesterol oxidation catalyzed by COD-B (Figure 1D), ROS levels were evaluated by detecting the DCF fluorescence intensity. As shown in Figure 4a, COD-B induced a dose-related increase in DCF fluorescence intensity, confirming that COD-B treatment did induce ROS generation. Cholesterol addition markedly elevated ROS levels, which was likely due to the oxidation of exogenous cholesterol and accumulation of hydrogen peroxide. The anti-apoptotic protein $\mathrm{Bcl}-2$ and proapoptotic protein Bax are closely related to ROS generation. We examined whether COD-B treatment could affect the expression of both proteins. As expected, COD-B treatment 


\section{A}

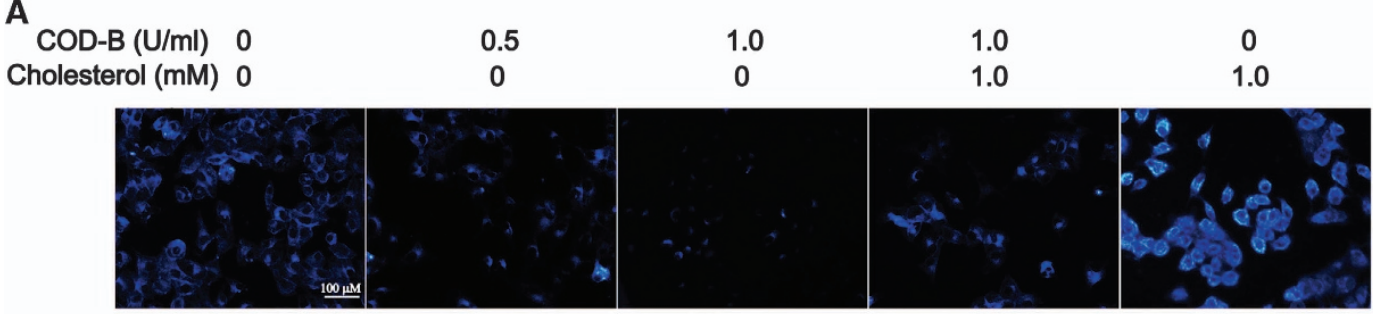

B

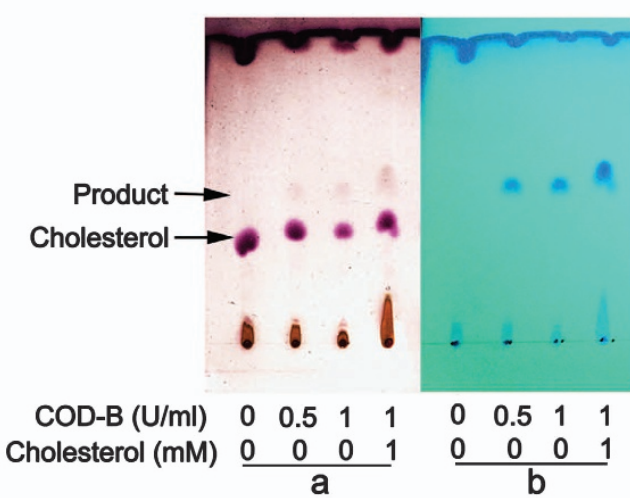

D

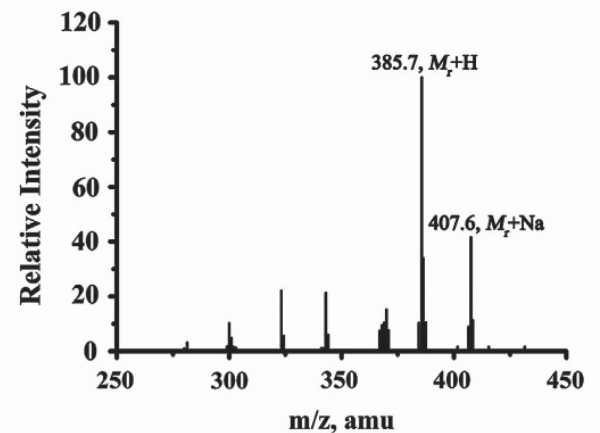

C

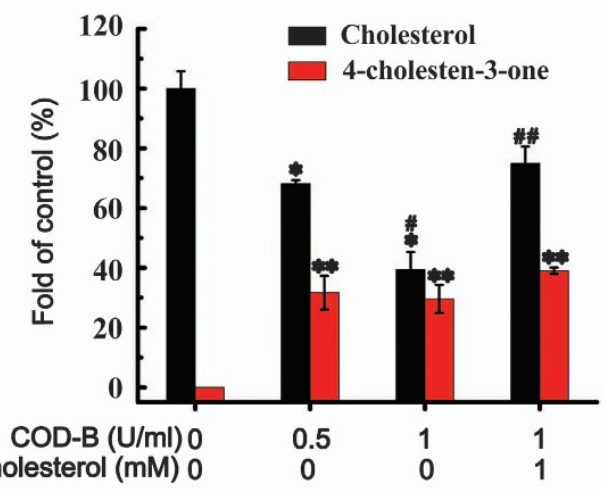

E

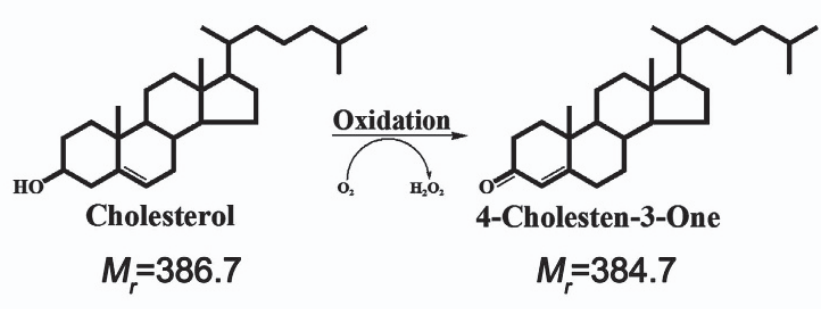

Figure 1 Effect of COD-B on membrane cholesterol in lung adenocarcinoma cells. A549 cells were treated with different concentration of COD-B or cholesterol for $6 \mathrm{~h}$. (A) Cholesterol staining by the sterol-binding probe filipin. (B) TLC analysis of membrane lipids extracted from A549 cells. (a) TLC plate was colorized by $10 \%$ sulfuric acid; (b) TLC plate was detected by UV irradiation. (C) Relative content of membrane cholesterol and oxidation product. (D) Mass spectrum analysis of the oxidation product. $M_{r}+\mathrm{H}$, represented molecular weight of product plus $\mathrm{H}^{+} ; M_{r}+\mathrm{Na}$, represented molecular weight of product plus $\mathrm{Na}^{+}$. (E) The cholesterol oxidation catalyzed by COD-B. $M_{F}$, represented molecular weight. Values are means of at least three independent experiments. ${ }^{*} P<0.05$ versus control group; ${ }^{*} P<0.01$ versus control group; ${ }^{\#} P<0.05$ versus the group treated with $0.5 \mathrm{U} / \mathrm{ml}$ COD-B; ${ }^{\# \#} P<0.01$ versus the group treated with $1.0 \mathrm{U} / \mathrm{ml}$ COD-B

induced the downregulation of BCL-2 and the upregulation of Bax (Figure 4b), which was promoted by cholesterol addition. Given that BCL-2 family members have important roles in mediating cytochrome $C($ Cyt $C$ ) release from mitochondria, we further tested the effect of COD-B on Cyt $C$ release. Figure $4 \mathrm{C}$ showed that COD-B treatment resulted in Cyt $C$ release from mitochondria to cytoplasma, which was enhanced by cholesterol addition. Because c-Jun NH2terminal kinase (JNK) and p38 are considered mediators of pro-apoptotic signaling in cells exposed to oxidative stress, we also tested whether COD-B influenced the activation of JNK and p38. The results authenticated that COD-B could induce phosphorylation of JNK and p38 (Figure 4d). Combined with COD-B-induced caspase-3 activation, we reasoned that the ROS-mitochondrial apoptotic pathway should be implicated in COD-B-induced apoptosis. Therefore, the activation of Akt and ERK1/2 was transiently induced by freshly generated hydrogen peroxide during the oxidation of cholesterol addition.

Subsequently, we tested the effect of catalase on the COD-B-induced signaling response. When cells were pretreated with catalase $(10 \mathrm{U} / \mathrm{ml})$ before adding COD-B and cholesterol, the phosphorylation of Akt and ERK $1 / 2$ remained attenuated compared with the group without catalase treatment (Figure 5a). Catalase pretreatment also partially blocked the activation of caspase-3. Furthermore, preincubation of cells with catalase led to a slight increase in cell viability compared with cells without catalase before COD-B treatment (Figure 5b). Inactive COD-B (incubation in boiling water for $5 \mathrm{~min}$ ) had little effect on cell viability in the presence of cholesterol and catalase. Because the hydrogen peroxideinduced phosphorylation of Akt and ERK $1 / 2$ peaked after a short time, we investigated the effect of COD-B on phosphorylation of Akt and ERK1/2 at short intervals. As shown in 


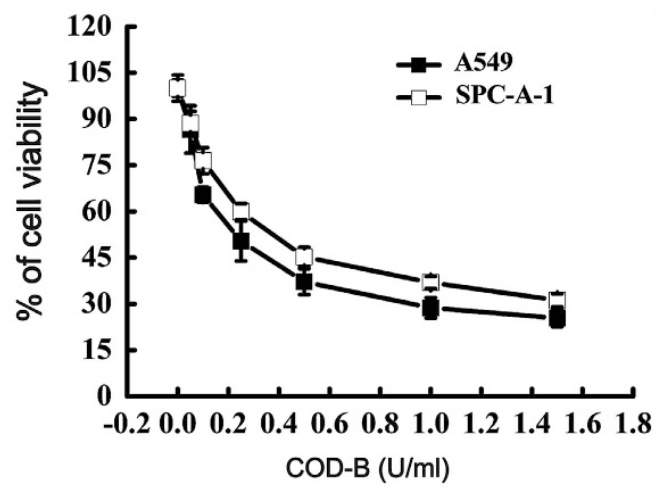

b

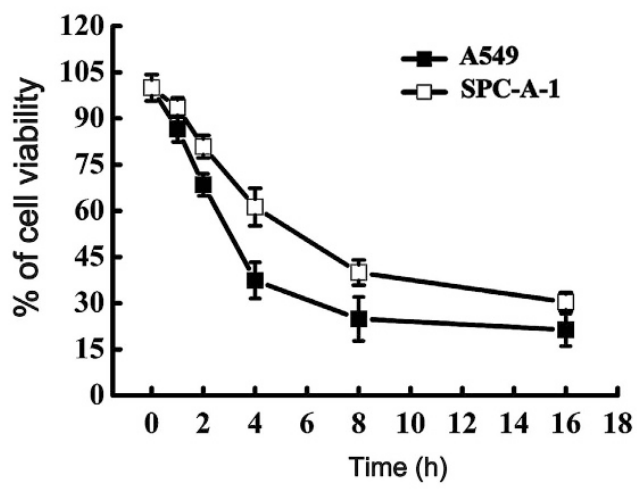

C

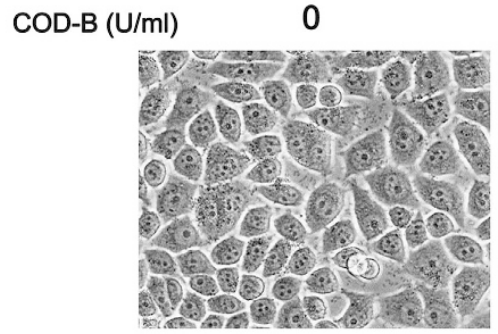

0.5

1.0
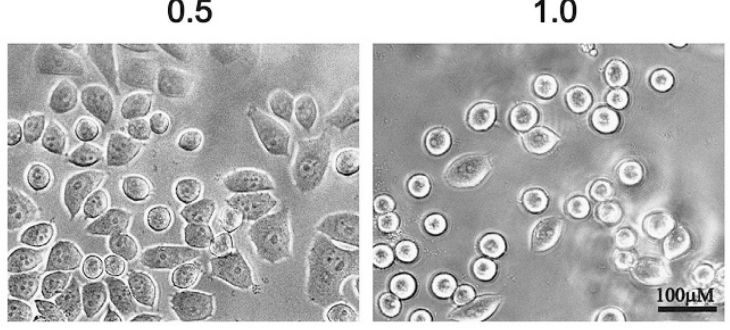

d

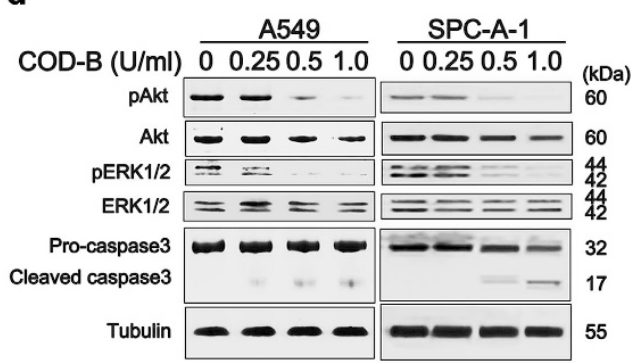

e

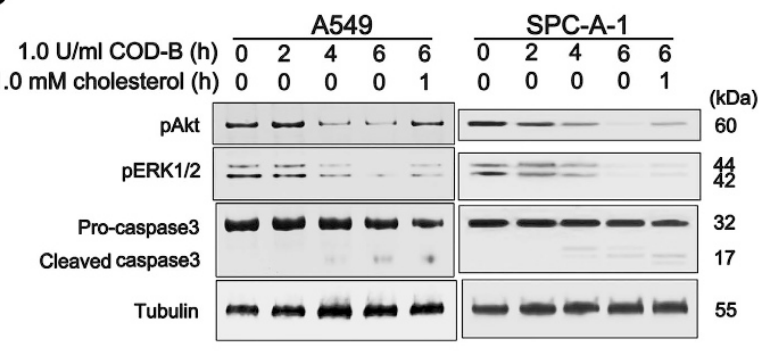

Figure 2 Effect of COD-B on cell viability, phosphorylation of Akt and ERK1/2 and caspase-3 activation. (a) Viability of serum-free A549 and SPC-A-1 cells treated with different concentrations of COD-B for $24 \mathrm{~h}$. (b) Viability of serum-free A549 and SPC-A-1 cells treated with $1.0 \mathrm{U} / \mathrm{ml}$ COD-B for the indicated time. (c) The microscopic images of serum-free A549 cells treated with the indicated concentrations of COD-B. (d) A549 and SPC-A-1 cells were incubated in serum-free medium with the indicated concentrations of COD-B overnight. Equal amounts of protein from each treatment were analyzed by western blotting. (e) A549 and SPC-A-1 cells were incubated in serum-free medium in the presence of $1.0 \mathrm{U} / \mathrm{ml} \mathrm{COD}-\mathrm{B}$ for the indicated time. Select COD-B-treated cells after $6 \mathrm{~h}$ were incubated with $1 \mathrm{mM}$ cholesterol for an additional $1 \mathrm{~h}$. Equal amounts of protein from each treatment were analyzed by western blotting. Values are means of at least three independent experiments

Figures $5 \mathrm{c}$ and $\mathrm{d}, \mathrm{COD}-\mathrm{B}$ induced the transient phosphorylation of Akt and ERK1/2 with peaks at $20 \mathrm{~min}$. After $240 \mathrm{~min}$ of exposure, the COD-B-induced phosphorylation of Akt and ERK $1 / 2$ returned to basal levels and decreased gradually to dephosphorylation. The addition of cholesterol could not block the transient activation. To verify the involvement of exogenous hydrogen peroxide in the COD-B-mediated signaling response, the catalase-pretreated cells were incubated with COD-B or COD-B/cholesterol for $20 \mathrm{~min}$, and then phosphorylation of Akt and ERK1/2 was examined. As shown in Figures $5 e$ and $f$, phosphorylation of Akt and ERK1/2 was significantly increased after exposure to $0.5 \mathrm{mM} \mathrm{H} 2 \mathrm{O} 2$ or $1.0 \mathrm{U} / \mathrm{ml} \mathrm{COD}-\mathrm{B}$ with $1 \mathrm{mM}$ or no cholesterol for $20 \mathrm{~min}$. Catalase treatment attenuated the phosphorylation of Akt and ERK $1 / 2$ induced by COD-B and also inhibited $\mathrm{H}_{2} \mathrm{O}_{2}$ induced activation of Akt and ERK1/2. These data indicated that exogenous $\mathrm{H}_{2} \mathrm{O}_{2}$ during oxidation of cholesterol indeed had a role in the COD-B-induced signaling response and apoptosis.

COD-B induced the reversible translocation of caveolin-1. Because caveolin-1 is believed to be involved in trafficking membrane cholesterol, we tested whether membrane cholesterol oxidation affected the location of caveolin-1. As shown in Figure 6, most of the caveolin-1 was assigned to the Triton $\mathrm{X}$-100-insoluble fraction in untreated cells. Immunofluorescent staining showed that caveolin-1 was mainly dispersed in the plasma membrane with a minor portion in the intercellular space in untreated cells. COD-B treatment induced the intercellular accumulation of caveolin-1, whereas the addition of cholesterol blocked the translocation. In accordance, we showed that caveolin-1 disappeared 
a

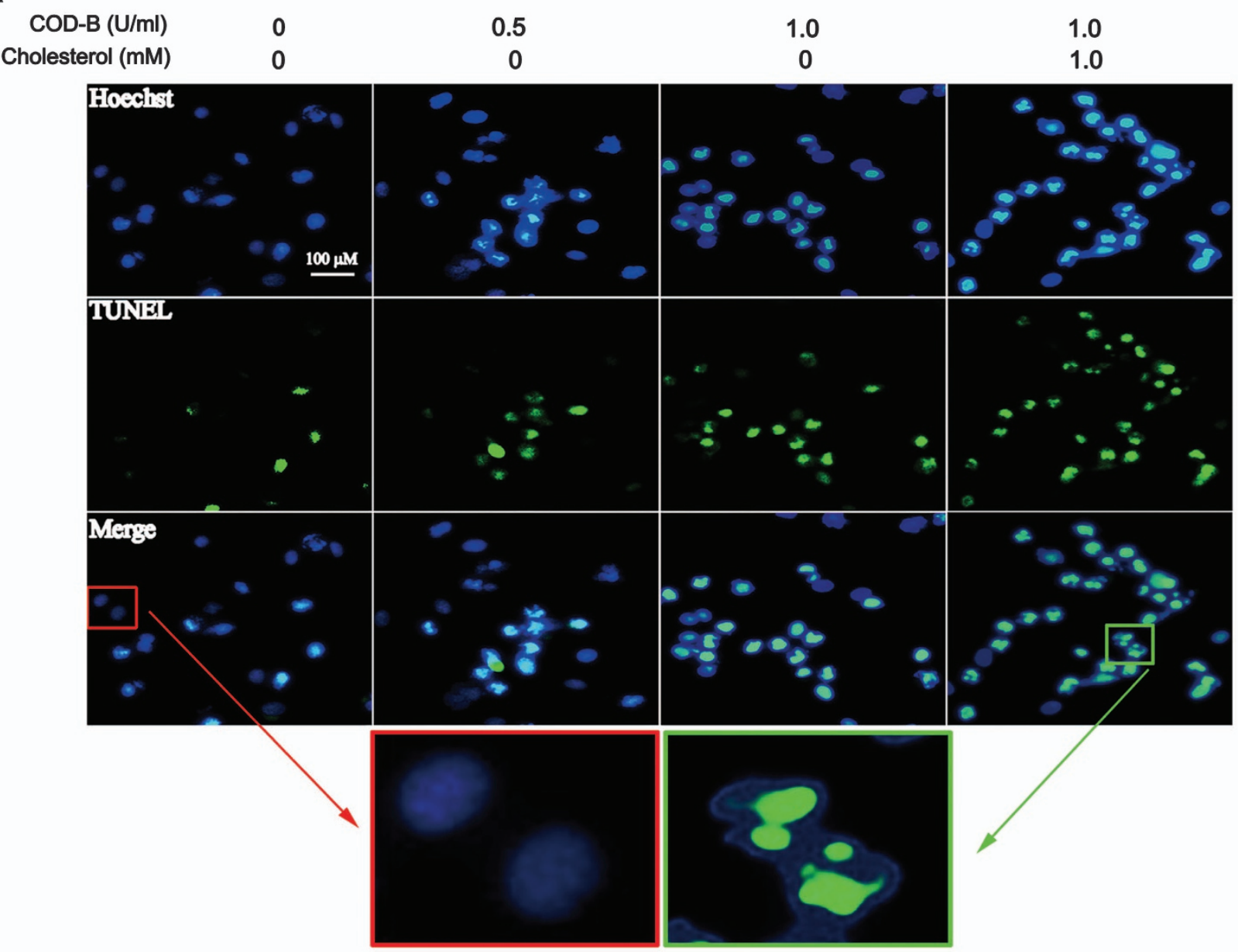

b

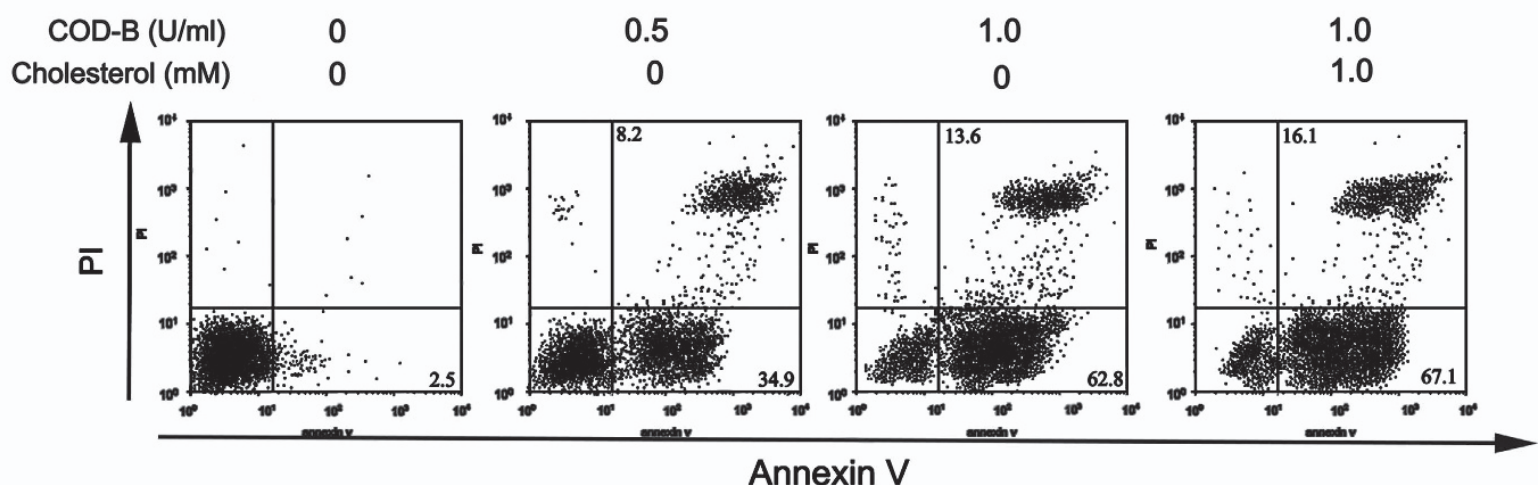

Figure 3 Analysis of apoptosis induced by COD-B. A549 cells were incubated in serum-free medium with the indicated concentrations of COD-B for $6 \mathrm{~h}$. Select COD-Btreated cells were incubated with $1 \mathrm{mM}$ cholesterol for an additional $1 \mathrm{~h}$. (a) The treated cells were assessed by Hoechst staining and TUNEL assay. (b) The treated cells were subjected to flow cytometer analysis. These experiments were repeated at least three times

from the Triton $X-100$-insoluble fraction in COD-B-treated cells by western blotting, which could be reversed by the addition of cholesterol. Interestingly, additional cholesterol induced the redistribution of most caveolin-1 into the plasma membrane but did not reduce the apoptotic rate.

COD-B induced apoptosis in xenograft tumors. To determine whether COD-B also induced apoptosis in vivo, we analyzed the effect of COD-B on growth and apoptosis of xenograft tumors. Compared with the control group, tumor volumes were decreased in the COD-Btreated group (Figures $7 \mathrm{a}$ and b), and the apoptotic percentage of tumor cells were significantly increased in the COD-B-treated group (Figure 7c), indicating that COD-B treatment inhibited tumors growth and induced tumors apoptosis in vivo. 
a

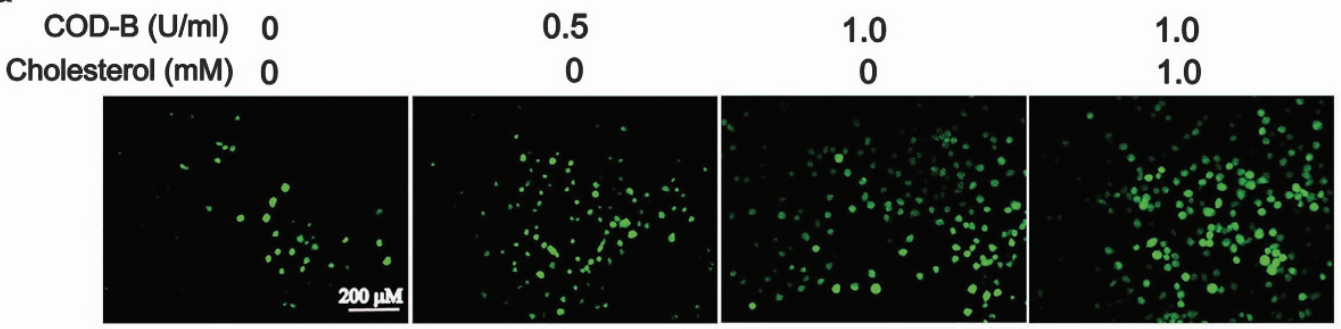

b

$\begin{array}{rllllll}1 \mathrm{U} / \mathrm{ml} \mathrm{COD-B}(\mathrm{h}) & 0 & 1 & 2 & 4 & 6 & 0 \\ 1 \mathrm{mM} \text { Cholesterol(h) } & 0 & 0 & 0 & 0 & 1 & 0\end{array}$

c
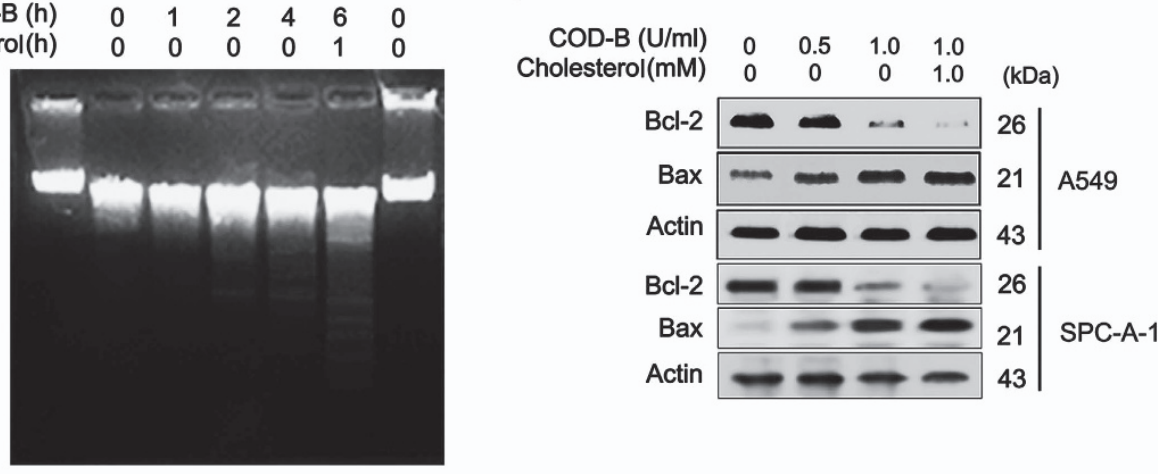

d

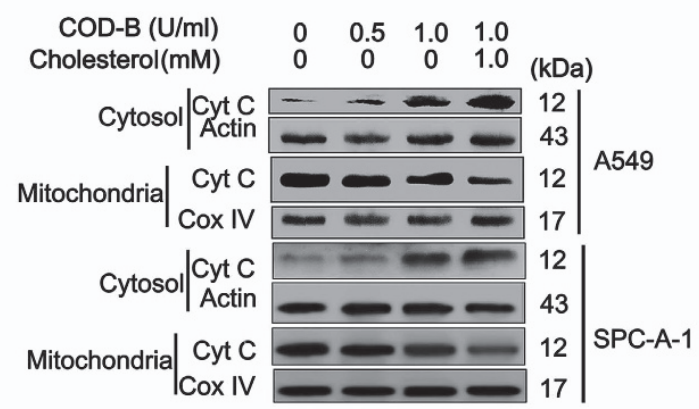

e

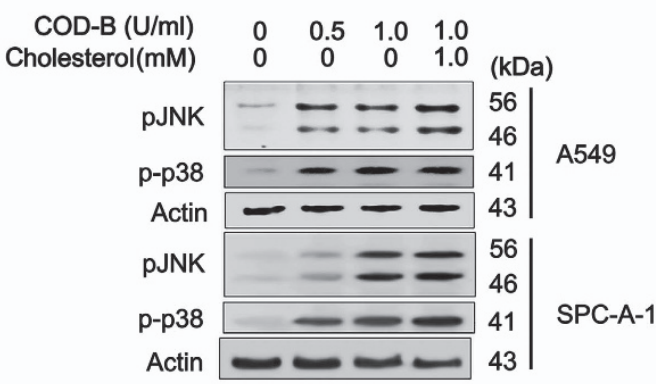

Figure 4 Effects of COD-B on ROS generation and expression of Bcl-2, Bax, Cyt C, JNK and p38. A549 and SPC-A-1 cells were incubated in serum-free medium with the indicated concentrations of COD-B for $6 \mathrm{~h}$. Select COD-B-treated cells were incubated with $1 \mathrm{mM}$ cholesterol for an additional $1 \mathrm{~h}$. (a) ROS generation was assessed by the oxidation-sensitive fluorescent probe (DCF-DA). (b) The profile of DNA from untreated and treated cells. (c) Effects of COD-B on the expression of Bcl-2 and Bax. Equal amounts of protein from each treatment were analyzed by western blotting. (d) Effects of COD-B on release of Cyt C from mitochondria to cytosol. (e) Effects of COD-B on phosphorylation of JNK and p38. All experiments were repeated at least three times

\section{Discussion}

COD has been utilized to detect the distribution of membrane cholesterol. ${ }^{35}$ For whole cells, only membrane cholesterol has responded to the oxidation of COD. ${ }^{35,36}$ However, because the action of COD is dependent on the microenvironment of cholesterol in the plasma membrane, such as the phospholipid composition, cholesterol content and ionic strength, this enzyme does not attack all cell types. ${ }^{35}$ Considering that the enzymatic properties of COD from diverse microorganisms are different, it is deduced that the reactivities of different CODs on plasma membranes significantly depend on their enzymatic properties, such as $\mathrm{pH}$ stability, temperature stability, optimal functional $\mathrm{pH}$ and temperature, stability in metal ionic buffers and $\mathrm{Km}$ values. ${ }^{36}$ Our previous study has demonstrated that COD-B, a COD isolated from Bordetella species, is very stable over a wide range of $\mathrm{pH}$ values and temperatures and exhibits optimal activity around a $\mathrm{pH}$ of 7.0 at $37^{\circ} \mathrm{C} .^{37}$ Moreover, metal ions can stimulate COD-B activity. ${ }^{37}$ Thus the enzymatic properties of COD-B, combined with elevated levels of membrane cholesterol in tumor cells, facilitate its action on the membrane of tumor cells. In the present study, our results demonstrated that exposure of A549 cells to COD-B could reduce the level of membrane cholesterol but did not obviously elevate the level of 4-cholesten-3-one (Figures $1 \mathrm{~A}$ and $\mathrm{C}$ ), which was consistent with previous reports. ${ }^{18,38}$ Therefore, it is likely that 4-cholesten-3-one was dislocated from the membrane due to loss of $3^{\prime}-\mathrm{OH}$.

The exposure to COD-B inhibited the growth of lung adenocarcinoma cells in vitro and in vivo. (Figures 2 and 7). Hoechst staining, TUNEL assay and flow cytometer analysis illustrated COD-B-induced apoptosis (Figure 3). Furthermore, DNA fragments' assay also indicated COD-B-induced 
a

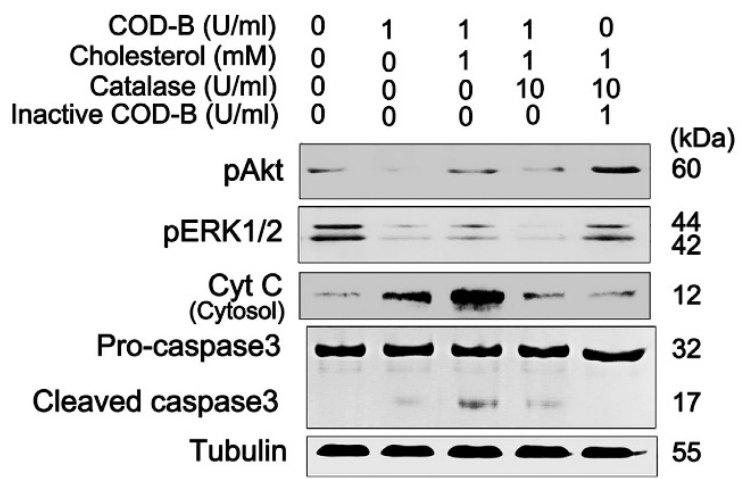

b

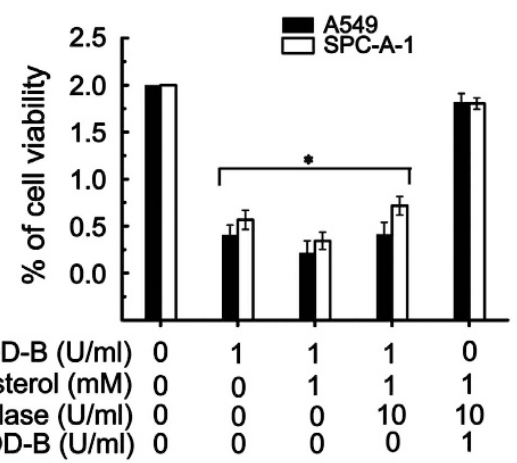

C
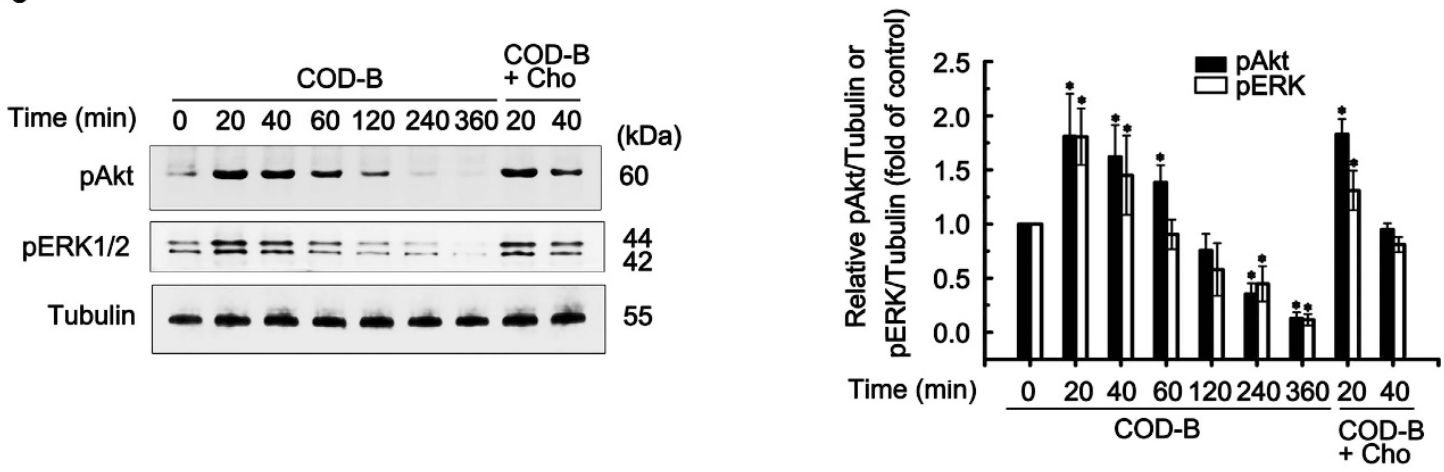

d
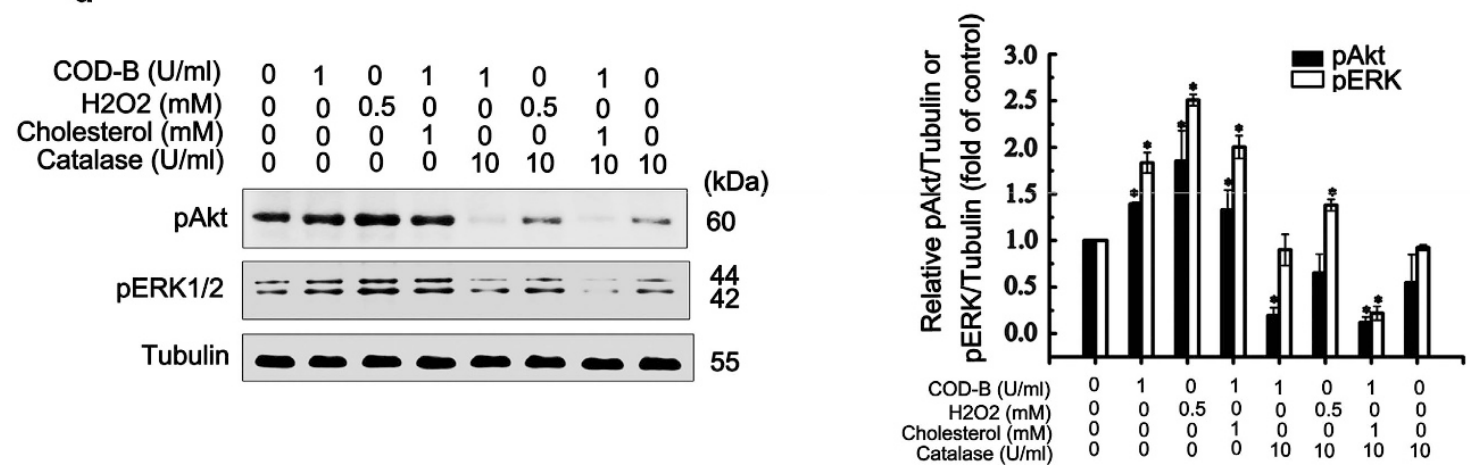

Figure 5 Effects of COD-B on different signaling pathways. (a) A549 cells were incubated in serum-free medium with or without 1.0 U/ml COD-B and 10 U/ml catalase for $6 \mathrm{~h}$. Select COD-B-treated cells were incubated with $1 \mathrm{mM}$ cholesterol for an additional $1 \mathrm{~h}$. Equal amounts of protein from each treatment were analyzed by western blotting. (b) Effects of different treatments as described in panel (a) on cell viability. ${ }^{*} P<0.05$ versus control group. (c) A549 cells were incubated in serum-free medium with $1.0 \mathrm{U} / \mathrm{ml} \mathrm{COD}-B$ for the indicated time. Select cells were incubated simultaneously with $1.0 \mathrm{U} / \mathrm{ml}$ COD-B and $1.0 \mathrm{mM}$ cholesterol for the indicated time. Equal amounts of protein from each treatment were analyzed by western blotting. Values are means of at least three independent experiments. ${ }^{*} P<0.05$, comparisons between groups treated for the indicated time with $1.0 \mathrm{U} / \mathrm{ml}$ COD-B in the presence or absence of cholesterol. (d) A549 cells were incubated in serum-free medium in the presence or absence of $1.0 \mathrm{U} / \mathrm{ml} \mathrm{COD}-\mathrm{B}, 1.0 \mathrm{mM}$ cholesterol, $10 \mathrm{U} / \mathrm{ml}$ catalase, or $0.5 \mathrm{mM} \mathrm{H}_{2} \mathrm{O}_{2}$ for $20 \mathrm{~min}$. Equal amounts of protein from each treatment were analyzed by western blotting. Values are means of at least three independent experiments. ${ }^{*} P<0.05$ versus control group

apoptosis (Figure 4b). Although COD has been explored in various cell types, such as $\mathrm{HaCaT}$ cells, ${ }^{9}$ hippocampal cells, ${ }^{37}$ Chinese hamster ovary cells, ${ }^{32}$ fibroblasts and kidney epithelial cells, ${ }^{38}$ the apoptotic mechanism is largely unclear. At present, the roles of membrane cholesterol in signal transduction have been widely investigated by cholesterol depletion or addition. Statins, as the HMG-CoA reductase inhibitor, effectively reduce the membrane cholesterol content and inhibit Akt phosphorylation, which results in cell apoptosis. ${ }^{23,25}$ Cholesterol depletion by $M \beta C D$ induces the inactivation of Akt and provokes the apoptosis of cells. ${ }^{24}$ However, cholesterol addition can reverse the effects of statins and $\mathrm{M} \beta \mathrm{CD}$. Our studies showed that $\mathrm{COD}-\mathrm{B}$ treatment for $6 \mathrm{~h}$ could inhibit Akt and ERK1/2 phosphorylation and could activate caspase-3 (Figure $2 \mathrm{~d}$ ) in a dose-dependent manner. Cholesterol addition could partially activate Akt and ERK1/2 but was not enough to prevent cells from undergoing apoptosis (Figures $2 \mathrm{e}$ and 3 ). Because $\mathrm{H}_{2} \mathrm{O}_{2}$ could rapidly 
a

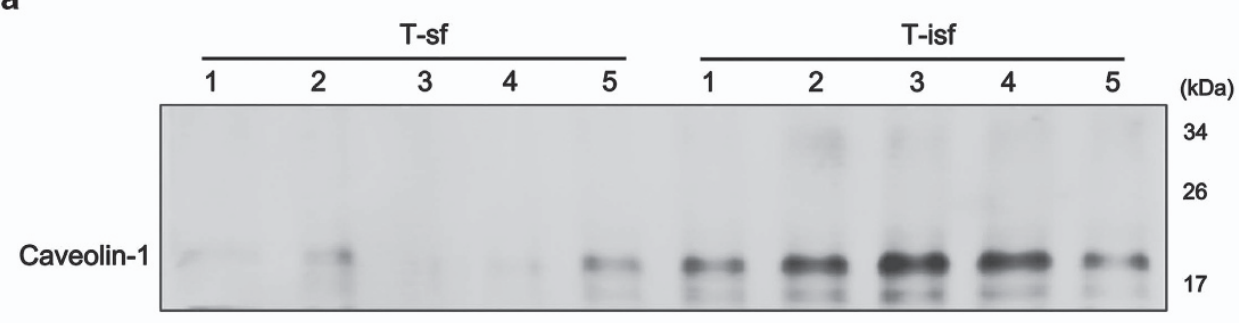

b

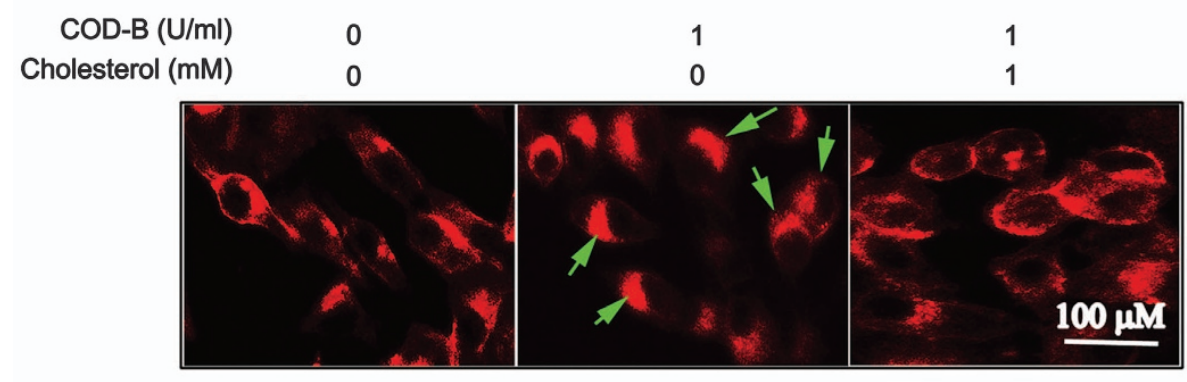

C

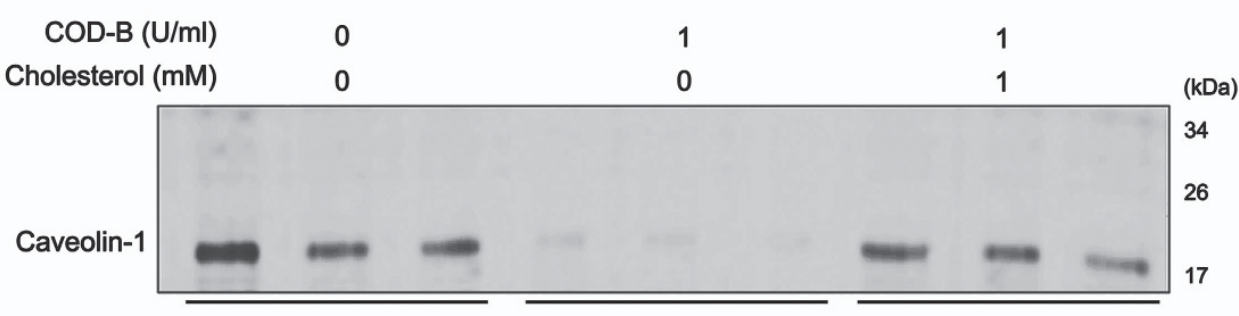

d

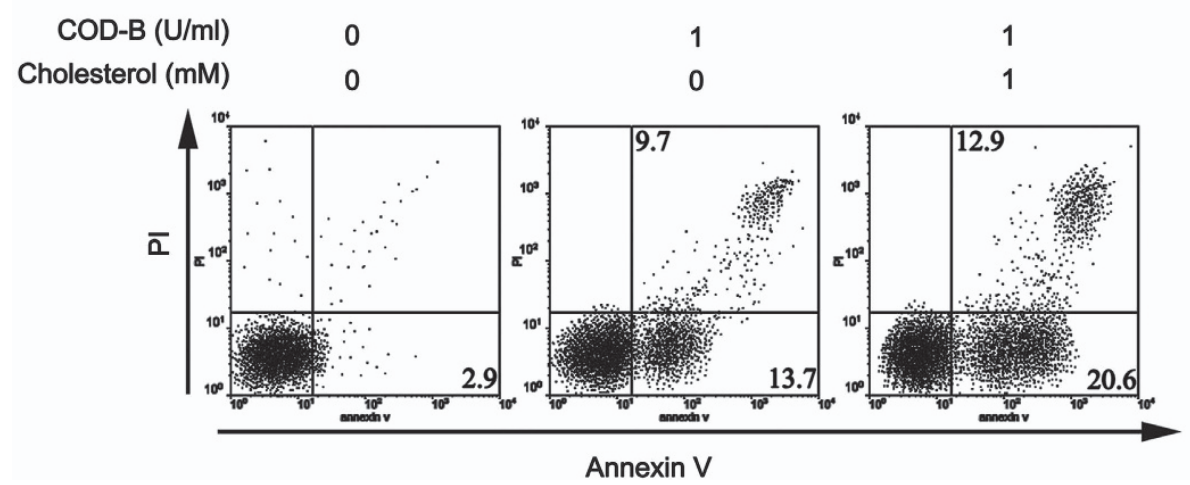

Figure 6 Effects of COD-B on caveolin-1 location. (a) Analysis of caveolin-1 location in untreated A549 cells by western blotting. Lanes 1-5 represent five repeated experiments; T-sf represents the Triton X-100-soluble fraction; T-isf represents the Triton X-100-insoluble fraction. (b) A549 cells were incubated in serum-free medium in the presence or absence of $1.0 \mathrm{U} / \mathrm{ml}$ COD-B or $1.0 \mathrm{mM}$ cholesterol for $1 \mathrm{~h}$. Cells were permeabilized and stained with an anti-caveolin-1 antibody. (c) Equal amounts of protein from T-isf from each treatment were analyzed by western blotting using anti-caveolin-1 antibody. (d) Cell apoptosis was assessed by flow cytometer analysis

induce transient phosphorylation of Akt and ERK1/2, ${ }^{39}$ it was speculated that the additional generation of $\mathrm{H}_{2} \mathrm{O}_{2}$ was involved in partial activation of Akt and ERK1/2. We first examined the levels of ROS during COD-B treatment with or without cholesterol. The results showed that elevated ROS levels depended on the dose of COD-B. Cholesterol addition promoted significant generation of ROS after COD-B treatment (Figure 4a). Along with the elevated ROS, COD-B promoted the generation of DNA fragments (Figure $4 b$ ), and
COD-B also induced Bcl-2 downregulation and Bax upregulation (Figure 4c). Furthermore, COD-B treatment promoted the release of Cyt $C$ from mitochondria to cytoplasma (Figure $4 d$ ). It has been well known that ROS mediates Cyt $C$ release from mitochondria. ${ }^{40}$ Correlating with the activation of JNK, p38 and caspase-3 (Figures 2d and 4e), the ROSmitochondrial signal pathway was implicated in COD-Binduced apoptosis. Therefore, the $\mathrm{H}_{2} \mathrm{O}_{2}$ produced, along with cholesterol oxidation, had a critical role in the signal 
a
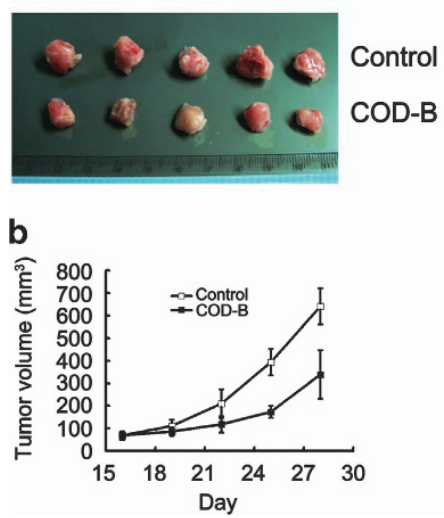

Figure 7 COD-B induced tumors' apoptosis in vivo. (a) Five xenograft tumors from control group and COD-B-treated group are shown. (b) COD-B treatment suppressed tumors; growth in vivo. (c) TUNEL assay revealed COD-B-induced apoptosis

transduction induced by COD-B. Hence, COD-B-mediated signal transduction might be a concurrent result of cholesterol depletion and $\mathrm{H}_{2} \mathrm{O}_{2}$ generation, which was different from the events induced by alone cholesterol depletion.

Further investigations were performed to ascertain the involvement of $\mathrm{H}_{2} \mathrm{O}_{2}$ in $\mathrm{COD}-\mathrm{B}$-mediated signal transduction by catalase pretreatment before cell exposure to COD-B. The results indicated that the cholesterol addition caused low levels of Akt and ERK1/2 phosphorylation in COD-B-treated cells. In contrast, catalase pretreatment decreased Akt and ERK1/2 phosphorylation and rescued a few cells from apoptosis induced by COD-B (Figures $5 \mathrm{a}$ and b), which suggested that the phosphorylation of Akt and ERK1/2 might be in response to fresh $\mathrm{H}_{2} \mathrm{O}_{2}$. We further tested the activation of Akt and ERK1/2 in the presence of COD-B without or with cholesterol at short intervals. Our results indicated that COD-B induced transient activation of Akt and ERK1/2 within $2 \mathrm{~h}$ and that there was a decrease in phosphorylation after $2 \mathrm{~h}$ (Figures $5 \mathrm{c}$ and d), which was similar to $\mathrm{H}_{2} \mathrm{O}_{2}$-induced phosphorylation. ${ }^{39}$ The simultaneous addition of COD-B and cholesterol for 20 or $40 \mathrm{~min}$ significantly provoked Akt and ERK1/2 phosphorylation, and transient activation of Akt and ERK1/2 seemed to rescue cells from apoptosis.

We found intriguing parallels between COD-B and $\mathrm{H}_{2} \mathrm{O}_{2}$ treatments, that is, both treatments caused transient phosphorylation of Akt and ERK1/2 in a short period of time, which could be abolished by catalase pretreatment (Figures $5 \mathrm{e}$ and $\mathrm{f}$ ). The difference was that the catalase pretreatment resulted in full dephosphorylation in the COD-B-treated groups, whereas catalase pretreatment only restored cells to basal phosphorylation in the $\mathrm{H}_{2} \mathrm{O}_{2}$-treated groups. These observations suggested that catalase reduced the $\mathrm{H}_{2} \mathrm{O}_{2}$ generated with cholesterol oxidation and eliminated $\mathrm{H}_{2} \mathrm{O}_{2}$ induced phosphorylation; however, catalase had little effect on the dephosphorylation induced by cholesterol depletion. Previous findings have revealed that cholesterol depletion led to the inactivation of Akt but the activation of ERK1/2. ${ }^{18,24}$ However, our results suggested that cholesterol oxidation affected phosphorylation of Akt and ERK1/2 in parallel, which should be a combined effect of cholesterol depletion and $\mathrm{H}_{2} \mathrm{O}_{2}$ generation.

It is well known that cholesterol depletion induces caveolae internalization, resulting in the inactivation of the Akt and ERK1/2 pathways. ${ }^{41,42}$ Caveolin-1 is an important structural protein of caveolae and is mainly distributed in the plasma membrane, with a small portion found in the Golgi apparatus. ${ }^{38}$ Upregulation of caveolin-1 has been reported in lung adenocarcinoma and prostate cancer, which are closely related with their metastatic and invasive capacities. ${ }^{43-46}$ In the present investigation, we found that cholesterol oxidation caused translocation of caveolin-1 from the plasma membrane to the cytoplasm (Figure 6). Cholesterol addition could reverse the translocation but did not prevent cell apoptosis, which suggested that cholesterol oxidation induced signal transduction and apoptosis independently of caveolin-1. Previous reports have proposed that lipid rafts are able to mediate signal transduction associated with tumor progression in the absence of caveolin-1. ${ }^{47}$ Cholesterol level has a more important role in cell survival than caveolin-1. ${ }^{21}$ Thus, combined with the reported literature, membrane cholesterol should be a potential therapeutic target. COD-B may be a promising therapeutic agent for tumors, especially for chemoresistant tumors, which might be due to elevated cholesterol levels and the action of COD-B dependent on the membrane cholesterol content. ${ }^{48,49}$

\section{Conclusions}

In summary, we have provided the evidence that COD-B, isolated from Bordetella species, oxidizes membrane cholesterol to 4-cholesten-3-one with the reduction of $\mathrm{O}_{2}$ to $\mathrm{H}_{2} \mathrm{O}_{2}$, which results in a decrease of cholesterol content and an increase of ROS levels. The events induced by COD-B lead to cell apoptosis by inactivation of the Akt and ERK1/2 pathway as well as activation of caspase-3. The elevated ROS levels also contribute to cell apoptosis by JNK phosphorylation and the imbalance between Bax and $\mathrm{Bcl}-2$, despite the initial transient phosphorylation of Akt and ERK1/2 that responded to oxidative stress to rescue the cells from apoptosis (Figure 8). COD-B also oxidizes exogenous cholesterol to 4-cholesten-3-one and $\mathrm{H}_{2} \mathrm{O}_{2}$. Therefore, cholesterol addition is not able to prevent the COD-B-induced apoptosis. These findings suggest that COD-B may be a promising therapeutic agent for cancer by targeting membrane cholesterol.

\section{Materials and methods}

Antibodies and reagents. The following monoclonal antibodies and polyclonal antibodies (pAbs) were used: anti-Akt (pAb) and anti-Bcl-2 (pAb) were from Anbo Biotechnology Company (San Francisco, CA, USA); anti-phospho-Akt (Ser473, pAb), anti-phospho-ERK1/2 (pAb), anti-caspase-3 (pAb), anti-Bax (pAb), anti-caveolin-1 (pAb), anti-phospho-JNK1/2 (pAb), anti-beta-actin (pAb) and anti-alpha-tubulin $(\mathrm{pAb})$ were purchased from Abcam (Cambridge, MA, USA). RIPA lysis buffer was purchased from Cell Signaling Technology (Beverly, MA, USA). The In Situ Cell Death Detection Kit was purchased from Roche Applied Science (Shanghai, China). 3-(4,5-Dimethyl thiazol-2-yl)-2,5-diphenyl tetrazolium bromide (MTT), $2^{\prime}, 7^{\prime}$-dichlorofluorescin diacetate (DCF-DA) and water-soluble cholesterol were purchased from Sigma Chemical Co. (St. Louis, MO, USA).

Cell culture. The human lung adenocarcinoma cell line A549 and SPC-A-1 were purchased from American Type Culture Collection (Manassas, VA, USA). Cells were cultured in Dulbecco's modified Eagle's medium (Hyclone, Logan, UT, USA) 


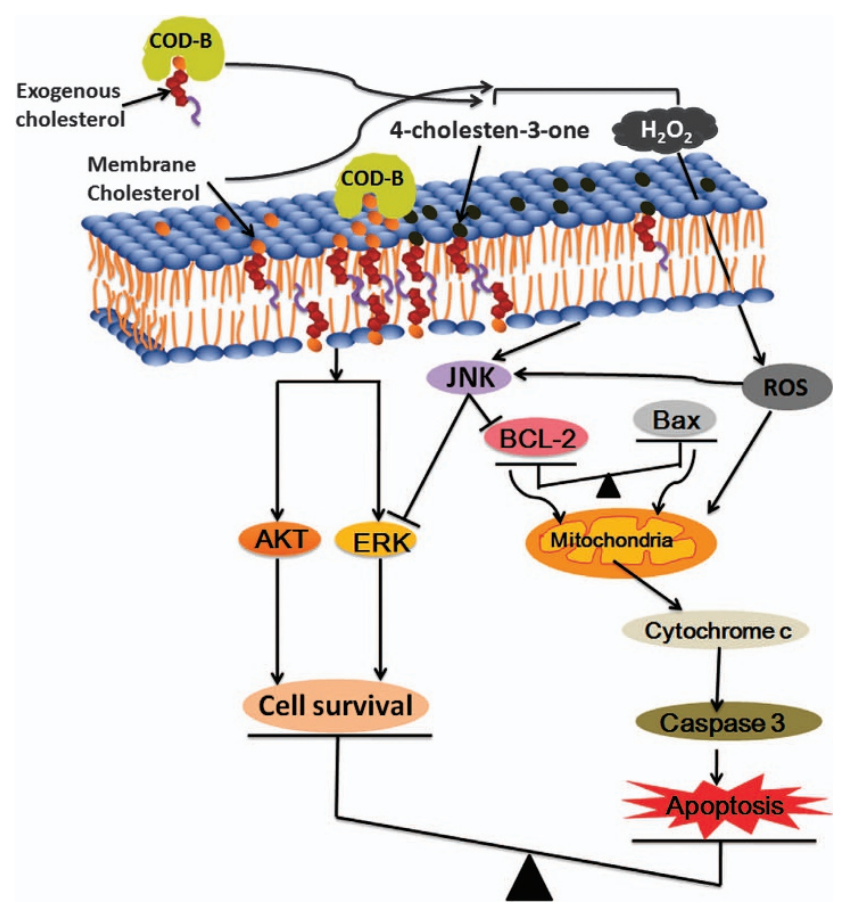

Figure 8 Summary diagram of the signal pathways likely involved in COD-B-induced apoptosis. COD-B oxidizes membrane or exogenous cholesterol to 4-cholesten-3-one and $\mathrm{H}_{2} \mathrm{O}_{2}$, transiently activating Akt and ERK1/2 to rescue the cells from apoptosis. Prolonged exposure to COD-B results in dephosphorylation of Akt and ERK1/2 and ROS generation. The elevated ROS contributes to JNK and $\mathrm{p} 38$ phosphorylation, upregulation of Bax, downregulation of $\mathrm{BCl}-2$ and $\mathrm{Cyt}$ $C$ release. Finally, caspase- 3 is activated, leading to cell apoptosis

supplemented with $10 \%$ fetal bovine serum (Hyclone), $100 \mathrm{U} / \mathrm{ml}$ penicillin, $100 \mathrm{U} / \mathrm{ml}$ streptomycin and $0.03 \%$ L-glutamine at $37^{\circ} \mathrm{C}$ in $5 \% \mathrm{CO}_{2}$.

COD treatments. COD from Bordetella sp. B4 (COD-B) was purified as previously described. ${ }^{37}$ When the cells were grown to approximately $50 \%$ confluence, the medium was changed to serum-free medium for $4 \mathrm{~h}$ before COD-B treatments. COD-B was diluted with fresh serum-free medium at the indicated concentration. The cells were washed with fresh serum-free medium and immediately exposed to various concentrations of COD-B for $24 \mathrm{~h}$. The cells were also cultured in serum-free medium containing $1.0 \mathrm{U} / \mathrm{ml}$ COD-B for the indicated time. To examine whether cholesterol oxidation could be reversed by the addition of cholesterol, some cells that had been treated with COD-B for $6 \mathrm{~h}$ were incubated with $1 \mathrm{mM}$ cholesterol for an additional $1 \mathrm{~h}$. One unit of COD-B (1 U) was defined as the amount of enzyme that converted $1 \mu \mathrm{mol}$ cholesterol into cholest-4-en-3-one per minute at $37^{\circ} \mathrm{C}$.

The analysis of cholesterol and oxidation products. The treated cells were rinsed with phosphate-buffered saline (PBS) and fixed with $4 \%$ paraformaldehyde for $30 \mathrm{~min}$. After rinsing three times, the cells were incubated with $100 \mu \mathrm{g}$ filipin for $1 \mathrm{~h}$ and detected by a fluorescence microscope (LEICA DMIRE2, Bensheim, Germany).

The preparation of the A549 cell membranes was performed as previously described. ${ }^{31}$ Briefly, cells $\left(1 \sim 2 \times 10^{8}\right)$ were scraped into the buffer (20 mM Tris, $250 \mathrm{mM}$ sucrose, $1.0 \mathrm{mM}$ EDTA, $10 \mathrm{mM}$ iodoacetamide, $200 \mu \mathrm{M}$ PMSF, $1 \mathrm{mM}$ DTT, $10 \mathrm{mg} / \mathrm{ml}$ aprotinin, $10 \mathrm{mg} / \mathrm{ml}$ leupeptin and $0.02 \%$ sodium azide, $\mathrm{pH}$ 7.4). The cell suspensions were sonicated on ice. After centrifugation at $100000 \times g$ for $1 \mathrm{~h}$, the membrane fraction pellet was separated from the cytosolic fraction and washed three times with the same buffer. The membrane pellet was mixed with chloroform and methanol $(2: 1, \mathrm{v} / \mathrm{v})$. After sonication, the chloroform layer extract was spotted onto a thin layer chromatography plate $\left(\mathrm{GF}_{254}, 0.25-\mathrm{mM}\right.$ thickness, $\left.10 \times 10 \mathrm{~cm}^{2}\right)$. The plate was developed in dichloromethane:petroleum ether: ethyl acetate $(7.7: 2: 0.3)$, visualized by UV irradiation and sprayed with $10 \%$ sulfuric acid.
The spot corresponding to the oxidation product was scraped from the plate and then redissolved in methanol for MS analysis.

Cell viability. The cells were seeded at $1 \times 10^{4}$ in $100 \mu \mathrm{l}$ culture medium in a 96well microplate. After treatment, MTT solution $(5 \mathrm{mg} / \mathrm{ml})$ was added and incubated together for $4 \mathrm{~h}$. The produced formazan was dissolved in DMSO and measured at the dual wavelength of $570 \mathrm{~nm}$ and $650 \mathrm{~nm}$ using SpectraMax M2 (MDC, CA, USA).

Analysis of apoptosis. Cell apoptosis was assessed by Hoechst staining, the TUNEL assay and flow cytometer analysis. The TUNEL assay was performed using the In Situ Cell Death Detection Kit (Roche). Briefly, cells were washed with cold PBS and fixed in $4 \%$ paraformaldehyde for $30 \mathrm{~min}$. The TUNEL reaction mixture was prepared immediately and incubated with cells for $1 \mathrm{~h}$. After rinsing three times with PBS, Hoechst33258 $(10 \mathrm{mg} / \mathrm{ml})$ was added and incubated for $20 \mathrm{~min}$. Apoptosed cells were detected under a fluorescence microscope (LEICA DMIRE2).

Flow cytometer analysis was applied to further determine the occurrence of apoptosis. The cells in the supernatant were collected and combined with digested cells using trypsin without EDTA and phenol red at $37^{\circ} \mathrm{C}$. After rinsing one time with PBS, cells were incubated with fluorescein isothiocyanate-conjugated annexin-V reagent $(2.5 \mathrm{mg} / \mathrm{ml})$ and $\mathrm{PI}(5 \mathrm{mg} / \mathrm{ml})$ in binding buffer followed by flow cytometer analysis.

DNA fragmentation assay. The cell were treated with $1 \mathrm{U} / \mathrm{ml}$ COD-B for the indicated time in the presence or absence of $1 \mathrm{mM}$ cholesterol. The treated cells were lysed in the buffer containing $10 \mathrm{mM}$ Tris- $\mathrm{HCl}(\mathrm{pH} 7.4), 10 \mathrm{mM}$ EDTA, $0.5 \%$ TritonX-100 and RNase A $(400 \mu \mathrm{g} / \mathrm{ml})$ for $30 \mathrm{~min}$ at $37^{\circ} \mathrm{C}$, followed by incubation with proteinase $\mathrm{K}(400 \mu \mathrm{g} / \mathrm{ml})$ for $1 \mathrm{~h}$ at $50^{\circ} \mathrm{C}$. The lysates were collected by centrifugation at $14000 \times g$ for $10 \mathrm{~min}$. The fragmented DNA in the supernatant was further extracted with phenol/chloroform/isoamyl alcohol $(25: 24: 1, v / v / v)$ and analyzed by agarose electrophoresis.

Protein extraction. The treated cells were harvested and resuspended in RIPA lysis buffer (Thermo, Waltham, MA, USA), which was incubated in ice for $30 \mathrm{~min}$. After centrifugation at $10000 \times g$ for $20 \mathrm{~min}$, the supernatant was collected as the total protein. Cytosol and mitochondria protein were isolated using cytoplasmic and mitochondrial protein extraction kit (Sangon Biotech, Shanghai, China) according to the manufacturer's protocol. Briefly, cells were collected by centrifugation at $800 \times g$ for $5 \mathrm{~min}$. After being washed with PBS, the cell pellets were resuspended in $1.0 \mathrm{ml}$ cytoplasmic extraction buffer and homogenized in a precooling Dounce homogenizer (Active Motif, Carlsbad, CA, USA), followed by vigorous shock for $15 \mathrm{~s}$ and incubation in ice for $15 \mathrm{~min}$. After centrifugation at $800 \times g$ for $10 \mathrm{~min}$, the supernatant was centrifuged again at $10000 \times g$ for $30 \mathrm{~min}$ at $4^{\circ} \mathrm{C}$. The supernatant was collected as a cytosol protein and the pellet was mitochondria fraction. The pellet was resuspended in $0.1-\mathrm{ml}$ cytoplasmic extraction buffer and vigorously shocked for $30 \mathrm{~s}$ at $4^{\circ} \mathrm{C}$. After centrifugation at $10000 \times g$ for $10 \mathrm{~min}$, the pellet was resuspended in $0.1-\mathrm{ml}$ mitochondria extraction buffer and incubated in ice for $30 \mathrm{~min}$. After centrifugation at $10000 \times \mathrm{g}$ for $10 \mathrm{~min}$, the supernatant was collected as mitochondria protein. The concentration of solubilized protein was determined using the bicinchoninic acid method.

Western blotting analysis. Equal amounts of proteins $(50 \mu \mathrm{g})$ were loaded and separated using $12 \%$ sodium dodecyl sulfate polyacrylamide gel electrophoresis. After separation, the proteins were transferred onto polyvinylidene fluoride membranes. The membranes were blocked overnight with $5 \% \mathrm{BSA}$ at $4{ }^{\circ} \mathrm{C}$ and then probed with the indicated primary antibodies overnight at $4{ }^{\circ} \mathrm{C}$. The membranes were washed three times with TBST followed by incubation with horseradish peroxidase-conjugated secondary antibodies for $1 \mathrm{~h}$ at room temperature. The labeled proteins were detected using the enhanced chemiluminescence method and quantified using Alpha Imager2200 (Cell Biosciences, Inc., Santa Clara, CA, USA).

Determination of ROS levels. The ROS level was assessed by the oxidation-sensitive fluorescent probe (DCF-DA). After treatment with COD-B, cells were washed with cold PBS and incubated in $10 \mu \mathrm{M}$ DCD-DA for $30 \mathrm{~min}$ at $37^{\circ} \mathrm{C}$. The fluorescence excitation was performed at $488 \mathrm{~nm}$, and the emission was detected at $525 \mathrm{~nm}$ using a fluorescence microscope (LEICA DMIRE2).

Immunofluorescence. Cells on cover slips were fixed with 4\% paraformaldehyde in PBS for 10 min, permeabilized with $0.2 \%$ Triton X-100 in PBS for 
$5 \mathrm{~min}$ and blocked with $2 \% \mathrm{BSA}$ in PBS for $1 \mathrm{~h}$ at room temperature. After washing with PBST three times, cells were incubated with anti-caveolin-1 $(1 \mu \mathrm{g} / \mathrm{ml})$ overnight at $4{ }^{\circ} \mathrm{C}$. Then, cells were washed gently with PBST for 10 times and were incubated with Texas red-conjugated secondary antibody IgG for $1 \mathrm{~h}$. After washing with PBST for five times, cells were detected under a fluorescence microscope (LEICA DMIRE2).

Analysis of caveolin-1. The isolation of caveolin-1 was performed as previously described. ${ }^{32}$ Briefly, cells $\left(1 \sim 2 \times 10^{8}\right)$ were washed with PBS and harvested and incubated in MBS buffer (MBS, $25 \mathrm{mM}$ 2-( $N$-morpholino)ethanesulfonic acid, pH 6.5, $150 \mathrm{mM} \mathrm{NaCl}, 1 \mathrm{mM}$ EDTA, $1 \mathrm{mM}$ PMSF) containing $1 \%$ Triton X-100 on ice for $30 \mathrm{~min}$. After thorough homogenization and centrifugation at $16000 \times g$, supernatants were obtained as Triton-soluble fractions. Insoluble pellets were resuspended in MBS buffer with $1 \%$ Triton X-100. After sonication on ice, Triton-insoluble fractions were obtained. Caveolin-1 in Triton-soluble and -insoluble fractions was analyzed by western blotting. Moreover, caveolin-1 in live cells was also detected by immunofluorescence.

Mice xenograft models. Male 6-week-old BALB/c nude mice were purchased from Weitonglihua Animal Center (Beijing, China) and maintained under specific pathogen-free conditions in the animal facility. All procedures were approved by the Institutional Laboratory Animal Care and Use Committee at Shandong Provincial hospital. A total of $10^{6}$ A549 cells in $100 \mu$ PBS were injected into both flanks of mice. Long $(L)$ and short axes $(S)$ of tumors were measured by vernier caliper, and the tumor volumes were calculated as follows: $V=\left(L \times S^{2}\right) \times \pi / 6{ }^{50}$ When the tumor volume reached approximately $60 \mathrm{mM}^{3}$, mice were randomized to two parts. Thereafter, COD-B ( $5 \mathrm{U}$, q3 days) was injected in tumor, and the mice in control group were injected with PBS. After treatment for 2 weeks, the mice were killed, and the tumors were removed for histological analysis.

Statistical analysis. Statistical analysis was performed using the SPSS software package (SPSS 18.0, Chicago, IL, USA). The results were presented as the mean \pm S.E. of at least three independent experiments. The comparisons between the two groups were performed by Student's $t$-test. The comparisons among multiple groups were performed by one-way ANOVA. $P<0.05$ was considered to be statistically significant.

\section{Conflict of Interest}

The authors declare no conflict of interest.

Acknowledgements. This work was supported by the National Natural Science Foundation of China (Grant No.81201778; 81100417) and the Promotive research fund for excellent young and middle-aged scientists of Shandong Province (Grant No.BS2011SW036).

\section{Author contributions}

YLL and $L G$ designed the experiments; JLL, GZX and YLL performed the experiments; ML, YZ, HL, MY, YY and SX performed data analysis; and JLL, GZX and YLL wrote the paper.

1. Simons K, Ikonen E. How cells handle cholesterol. Science 2000; 290: 1721-1726.

2. Ikonen E. Cellular cholesterol trafficking and compartmentalization. Nat Rev Mol Cell Biol 2008; 9: 125-138.

3. Balse E, Steele DF, Abriel H, Coulombe A, Fedida D, Hatem SN. Dynamic of ion channe expression at the plasma membrane of cardiomyocytes. Physiol Rev 2012; 92: 1317-1358.

4. Lingwood D, Simons K. Lipid rafts as a membrane-organizing principle. Science 2010; 327: $46-50$.

5. Simons K, Toomre D. Lipid rafts and signal transduction. Nat Rev Mol Cell Biol 2000; 1: 31-39.

6. Galbiati F, Razani B, Lisanti MP. Emerging themes in lipid rafts and caveolae. Cell 2001; 106: 403-411.

7. Silvius JR. Role of cholesterol in lipid raft formation: lessons from lipid model systems Biochim Biophys Acta 2003; 1610: 174-183.

8. Epand RM. Proteins and cholesterol-rich domains. Biochim Biophys Acta 2008; 1778 1576-1582.

9. Park EK, Park MJ, Lee SH, Li YC, Kim J, Lee JS et al. Cholesterol depletion induces anoikis-like apoptosis via FAK down-regulation and caveolae internalization. $J$ Pathol 2009; 218: 337-349.

10. Das M, Das DK. Lipid raft in cardiac health and disease. Curr Cardiol Rev 2009; 5: 105-111.
11. Krycer JR, Brown AJ. Cholesterol accumulation in prostate cancer: a classic observation from a modern perspective. Biochim Biophys Acta 2013; 1835: 219-229.

12. Magura L, Blanchard R, Hope B, Beal JR, Schwartz GG, Sahmoun AE. Hypercholesterolemia and prostate cancer: a hospital-based case-control study. Cancer Causes Control 2008; 19: 1259-1266

13. Bélanger MM, Gaudreau M, Roussel E, Couet J. Role of caveolin-1 in etoposide resistance development in A549 lung cancer cells. Cancer Biol Ther 2004; 3: 954-959.

14. Li HY, Appelbaum FR, Willman CL, Zager RA, Banker DE. Cholesterol-modulating agents kill acute myeloid leukemia cells and sensitize them to therapeutics by blocking adaptive cholesterol responses. Blood 2003; 101: 3628-3634.

15. Todor IN, Lukyanova NY, Chekhun VF. The lipid content of cisplatin- and doxorubicinresistant MCF-7 human breast cancer cells. Exp Oncol 2012; 34: 97-100.

16. Staubach S, Hanisch FG. Lipid rafts: signaling and sorting platforms of cells and their roles in cancer. Expert Rev Proteomics 2011; 8: 263-277.

17. Loeper S, Asa SL, Ezzat S. Ikaros modulates cholesterol uptake: a link between tumor suppression and differentiation. Cancer Res 2008; 68: 3715-3723.

18. Lee SH, Koo KH, Park JW, Kim HJ, Ye SK, Park JB et al. HIF-1 is induced via EGFR activation and mediates resistance to anoikis-like cell death under lipid rafts/caveolaedisrupting stress. Carcinogenesis 2009; 30: 1997-2004.

19. Jeon JH, Kim SK, Kim HJ, Chang J, Ahn CM, Chang YS. Lipid raft modulation inhibits NSCLC cell migration through delocalization of the focal adhesion complex. Lung Cancer 2010; 69: 165-171.

20. Schley PD, Brindley DN, Field CJ. (n-3) PUFA alter raft lipid composition and decrease epidermal growth factor receptor levels in lipid rafts of human breast cancer cells. J Nutr 2007; 137: 548-553.

21. Li YC, Park MJ, Ye SK, Kim CW, Kim YN. Elevated levels of cholesterol-rich lipid rafts in cancer cells are correlated with apoptosis sensitivity induced by cholesterol-depleting agents. Am J Pathol 2006; 168: 1107-1118.

22. Oh HY, Lee EJ, Yoon S, Chung BH, Cho KS, Hong SJ. Cholesterol level of lipid raft microdomains regulates apoptotic cell death in prostate cancer cells through EGFR-mediated Akt and ERK1/2 signal transduction. Prostate 2007; 67: 1061-1069.

23. Skaletz-Rorowski A, Lutchman M, Kureishi Y, Lefer DJ, Faust JR, Walsh K. HMG-CoA reductase inhibitors promote cholesterol-dependent Akt/PKB translocation to membrane domains in endothelial cells. Cardiovasc Res 2003; 57: 253-264.

24. Calay D, Vind-Kezunovic D, Frankart A, Lambert S, Poumay Y, Gniadecki R. Inhibition of Akt signaling by exclusion from lipid rafts in normal and transformed epidermal keratinocytes. J Invest Dermatol 2010; 130: 1136-1145.

25. Spampanato C, De Maria S, Sarnataro M, Giordano E, Zanfardino M, Baiano S et al. Simvastatin inhibits cancer cell growth by inducing apoptosis correlated to activation of Bax and down-regulation of BCL-2 gene expression. Int J Oncol 2012; 40: 935-941.

26. Pitha J, Irie T, Sklar PB, Nye JS. Drug solubilizers to aid pharmacologists: amorphous cyclodextrin derivatives. Life Sci 1988; 43: 493-502.

27. Schnitzer JE, Oh P, Pinney E, Allard J. Filipin-sensitive caveolae-mediated transport in endothelium: reduced transcytosis, scavenger endocytosis, and capillary permeability of select macromolecules. J Cell Biol 1994; 127: 1217-1232.

28. Di Vizio D, Solomon KR, Freeman MR. Cholesterol and cholesterol-rich membranes in prostate cancer: an update. Tumori 2008; 94: 633-639.

29. Mendes MV, Recio E, Antón N, Guerra SM, Santos-Aberturas J, Martín JF et al. Cholesterol oxidases act as signaling proteins for the biosynthesis of the polyene macrolide pimaricin. Chem Biol 2007; 14: 279-290.

30. Cahuzac N, Baum W, Kirkin V, Conchonaud F, Wawrezinieck L, Marguet D et al. Fas ligand is localized to membrane rafts, where it displays increased cell death-inducing activity. Blood 2006; 107: 2384-2391.

31. Pucadyil TJ, Shrivastava S, Chattopadhyay A. Membrane cholesterol oxidation inhibits ligand binding function of hippocampal serotonin $(1 \mathrm{~A})$ receptors. Biochem Biophys Res Commun 2005; 331: 422-427.

32. Okamoto Y, Ninomiya H, Miwa S, Masaki T. Cholesterol oxidation switches the internalization pathway of endothelin receptor type A from caveolae to clathrin-coated pits in Chinese hamster ovary cells. J Biol Chem 2000; 275: 6439-6446.

33. Vaux DL, Korsmeyer SJ. Cell death in development. Cell 1999; 96: 245-254.

34. Das M, Mukherjee SB, Shaha C. Hydrogen peroxide induces apoptosis-like death in Leishmania donovani promastigotes. J Cell Sci 2001; 114: 2461-2469.

35. Lange Y. Tracking cell cholesterol with cholesterol oxidase. J Lipid Res 1992; 33: 315-321.

36. MacLachlan J, Wotherspoon AT, Ansell RO, Brooks CJ. Cholesterol oxidase: sources, physical properties and analytical applications. J Steroid Biochem Mol Biol 2000; 72: 169-195.

37. Lin Y, Fu J, Song X. Purification and characterization of an extracellular cholesterol oxidase from a Bordetella species. Process Biochem 2010; 45: 1563-1569.

38. Smart EJ, Ying YS, Conrad PA, Anderson RG. Caveolin moves from caveolae to the Golgi apparatus in response to cholesterol oxidation. J Cell Biol 1994; 127: 1185-1197.

39. Yang B, Oo TN, Rizzo V. Lipid rafts mediate $\mathrm{H} 2 \mathrm{O} 2$ prosurvival effects in cultured endothelial cells. FASEB J 2006; 20: 1501-1513.

40. Autret A, Martin SJ. Emerging role for members of the Bcl-2 family in mitochondrial morphogenesis. Mol Cell 2009; 36: 355-363.

41. Lasserre R, Guo XJ, Conchonaud F, Hamon Y, Hawchar O, Bernard AM. Raft nanodomains contribute to Akt/PKB plasma membrane recruitment and activation. Nat Chem Biol 2008; 4: 538-547. 
42. Echarri A, Del Pozo MA. Caveolae internalization regulates integrin-dependent signaling pathways. Cell Cycle 2006; 5: 2179-2182.

43. Williams TM, Hassan GS, Li J, Cohen AW, Medina F, Frank PG. Caveolin-1 promotes tumor progression in an autochthonous mouse model of prostate cancer: genetic ablation of Cav-1 delays advanced prostate tumor development in tramp mice. J Biol Chem 2005; 280: 25134-25145.

44. Ho CC, Huang PH, Huang HY, Chen YH, Yang PC, Hsu SM. Up-regulated caveolin-1 accentuates the metastasis capability of lung adenocarcinoma by inducing filopodia formation. Am J Pathol 2002; 161: 1647-1656.

45. Yoo SH, Park YS, Kim HR, Sung SW, Kim JH, Shim YS. Expression of caveolin-1 is associated with poor prognosis of patients with squamous cell carcinoma of the lung Lung Cancer 2003; 42: 195-202.

46. Cassoni P, Daniele L, Maldi E, Righi L, Tavaglione V, Novello S. Caveolin-1 expression in lung carcinoma varies according to tumour histotype and is acquired de novo in brain metastases. Histopathology 2009; 55: 20-27.

47. Kim J, Adam RM, Solomon KR, Freeman MR. Involvement of cholesterol-rich lipid rafts in interleukin-6-induced neuroendocrine differentiation of $\mathrm{LNCaP}$ prostate cancer cells. Endocrinology 2004; 145: 613-619.

48. Maksumova L, Ohnishi K, Muratkhodjaev F, Zhang W, Pan L, Takeshita A. Increased sensitivity of multidrug-resistant myeloid leukemia cell lines to lovastatin. Leukemia 2000; 14: 1444-1450.
49. Dimitroulakos J, Yeger H. HMG-CoA reductase mediates the biological effects of retinoic acid on human neuroblastoma cells: lovastatin specifically targets P-glycoproteinexpressing cells. Nat Med 1996; 2: 326-333.

50. Huang J, Ni J, Liu K, Yu Y, Xie M, Kang R et al. HMGB1 promotes drug resistance in osteosarcoma. Cancer Res 2012; 72: 230-238.

cC) (i) $($ )

Cell Death and Disease is an open-access journal published by Nature Publishing Group. This work is licensed under a Creative Commons Attribution-NonCommercialNoDerivs 3.0 Unported License. The images or other third party material in this article are included in the article's Creative Commons license, unless indicated otherwise in the credit line; if the material is not included under the Creative Commons license, users will need to obtain permission from the license holder to reproduce the material. To view a copy of this license, visit http://creativecommons.org/licenses/ by-nc-nd/3.0/ 